a control group of 25 age-matched boys.

The ADHD+ group, with motor problems, showed deficits in grip-force control during the precision grip-lift task. They had difficulties in adapting motor output to target different weights, suggesting deficiencies in neuromotor control mechanisms, independent of ADHD symptoms. An overall high grip force output used by the ADHD+ group was a compensatory mechanism to increase grip stability, secondary to impaired tactile stimulation and sensory motor control. In the group with ADHD without motor problems, these deficits were less pronounced. (Pereira HS, Eliasson A-C, Forssberg H. Detrimental neural control of precision grip lifts in children with ADHD. Dev Med Child Neurol August 2000;42:545-553). (Respond: Heloisa S Pereira MD MSc, Motorik Lab, Astrid Lindgrens Children's Hospital, S 17776 Stockholm, Sweden).

COMMENT. Discrete deficits in motor performance, independent of core symptoms of inattention and hyperactivity, occur in children with ADHD complicated by motor problems. Subtypes of ADHD, with and without motor dysfunction, argue against the hypothesis of impaired motor control related to a common neural mechanism for ADHD.

The descriptive term, DAMP (deficiency of attention, motor control, and perception) has been introduced in Scandinavian countries to characterize children with ADHD complicated by motor and perceptual disorders. In the USA, DAMP is regarded as a combination of ADHD and DCD (developmental coordination disorder). Before the American Psychiatric Association introduced the symptom complexes of ADHD and $\mathrm{DCD}$, these patients were regarded as 'clumsy' or having "minimal brain dysfunction (MBD)," and having subtle neurologic abnormalities on examination. The reintroduction and grading of the neurologic findings in the diagnosis of children with ADHD would introduce some objectivity to the definition of the syndrome.

\title{
STIMULANT TREATMENT OF ADHD IN PUBLIC SCHOOLS
}

A statewide school survey, supervised by school nurses, was performed to determine the prevalence of stimulant medication administered to Maryland public school students for the treatment of attention deficit hyperactivity disorder (ADHD), and reported by Johns Hopkins University Medical Institutions, and the Maryland State Department of Education, Baltimore, MD.

Of 816,465 students surveyed, $20,050(2.46 \%)$ received methylphenidate and $3721(0.46 \%)$ received other medications for ADHD. Methylphenidate was the most common stimulant prescribed, and represented $84 \%$ of all drug treatment for ADHD. Amphetamines were used in $11 \%$, clonidine in $1.7 \%$, pemoline in $0.4 \%$, and tricyclic antidepressants in $0.4 \%$. The male:female ratio was $3.5: 1$ for children receiving medication in elementary schools, and $4.3: 1$ in secondary schools. White children were medicated twice as often as black and Hispanic students.

Almost $50 \%$ of children receiving methylphenidate had special education accommodations, and $8.3 \%$ were eligible for Section 504 services, having an impairment that limited their major life activities. Children with Individual Education Programs (IEP), a marker for special education, received medication 5.6 times more often than students in regular education $(8.7 \%$ of students compared to $1.55 \%$, respectively). In high school, students with IEP were 10 -fold more likely to be receiving methylphenidate in school than regular students. School-district rates of methylphenidate treatment varied 5-fold geographically, from a low of $1.18 \%$ to a high of $6.02 \%$. Geographic variability was influenced by race/ethnicity demographics, and by large ADHD clinics in some localities.

By medical specialty, pediatricians were the prescribers of 
methylphenidate in $63 \%$ of students, family practitioners in $17 \%$, psychiatrists, $11 \%$, and nurse practitioners in $3 \%$. When drugs other than methylphenidate were prescribed, the psychiatrist's role was increased to 29\%. (Safer DJ, Malever M. Stimulant treatment in Maryland Public Schools. Pediatrics September 2000;106:533-539). (Reprints: Daniel J Safer MD, 7702 Dunmanway, Baltimore, MD 21222).

COMMENT. In the authors' summary of their findings, the rate of medication, mainly methylphenidate, administered to students during school hours for ADHD in Maryland public schools was found to vary with several factors: 4-fold by gender (male:female ratio); 2-fold by ethnicity/race (white vs minority); 3-fold by school level (elementary vs high school); 6-fold by educational category (special vs regular); and 5 -fold by school district (highest vs lowest rate). Surveys of the prevalence of stimulant use in school age children must necessarily include all these variables. In addition, the specialty of the practitioner treating these patients must be considered, since the majority were supervised by pediatricians. The absence of the pediatric neurologist as a specialist for the treatment of ADHD in Maryland was disappointing.

\section{ABNORMAL CEREBRAL BLOOD FLOW IN CHILDHOOD AUTISM}

Brain-perfusion single photon emission computed tomography was used to determine regional cerebral blood flow (rCBF) in 23 children with infantile autism and 26 non-autistic controls, evaluated at the National Center of Neurology and Psychiatry, Kodaira City, Tokyo, Japan. Decreases in rCBF were identified in the bilateral insula, superior temporal gyri, and the left prefrontal cortices of autistic patients, compared to controls. Patients were divided into two clinical syndrome categories according to symptoms, and each was correlated with a specific pattern of perfusion in the limbic system and the medial prefrontal cortex. Category 1, with impairments in communication and social interaction, had altered perfusion in the medial prefrontal cortex and anterior cingulate gyrus, and category 2, with obsessive desire for sameness, had altered perfusion in the right medial temporal lobe. Perfusion abnormalities showed some relation to cognitive dysfunction, abnormal responses to sensory stimuli, and an obsessive desire for sameness. The patterns of perfusion point to possible locations of brain dysfunction that correlate with abnormal behavior in autistic individuals. (Ohnishi T, Matsuda H, Hashimoto T et al. Abnormal regional cerebral blood flow in childhood autism. Brain September 2000;123:1838-1844). (Respond: Dr Takashi Ohnishi, Department of Radiology, 4-1-1 Ogawa higashi, Kodaira City, Tokyo, Japan 187$0031)$.

COMMENT. Children with infantile autism have abnormalities of cerebral perfusion involving the insula, temporal cortex, medial prefrontal cortex, anterior cingulate gyrus and hippocampus. These perfusion abnormalities are related to the cognitive and behavioral abnormalities seen in autism, and correlate with specific locations of brain dysfunction.

The authors refer to a deficit in the theory of mind tasks (ToM), a widely accepted explanation for the cognitive disorders of communication, socialization, and imagination in autistic children. ToM is the ability to explain and predict the behavior of others in terms of their mental states, such as beliefs and desires. Studies show that most autistic subjects fail the so-called false-belief task, an indication of a central cognitive deficit. The ability to attribute mental states has been located in the medial prefrontal cortex, an area with altered cerebral blood flow in autism. The orbitofrontal cortex and amygdala have also been associated with the ToM and ability to attribute mental states (Baron-Cohen et al. 1999). 